Ann. Biol. anim. Bioch. Biophys., 1967, 7 (I) 105-108.

\title{
SÉPARATION DES ISOMÈRES DE LA PSEUDOURIDINE
}

\author{
G. PÉTRISSANT \\ Laboratoire de Physiologie de la Lactation, \\ Centre national de Recherches zootechniques, 78 -Jouy-en-Josas
}

Au cours de dosages de l'acide pseudouridylique dans les ARN de transfert, nous avons été amené à étudier le comportement des isomères de la pseudouridine dans les systèmes chromatographiques que nous utilisons couramment. Nous avons, dans ce but, analysé une préparation commerciale de pseudouridine purifiée, dont 1'hétérogénéité a été précédemment signalée (OFENGAND et SCHAEFER, I965). Nous rapportons brièvement les résultats de ce travail.

La pseudouridine (Calbiochem, !A grade, Lot $n^{r}$ 30259) est chromatographiée sur Dowex $1 \times 8$ minus 400 mesh. L'échangeur est utilisé sous forme formate et

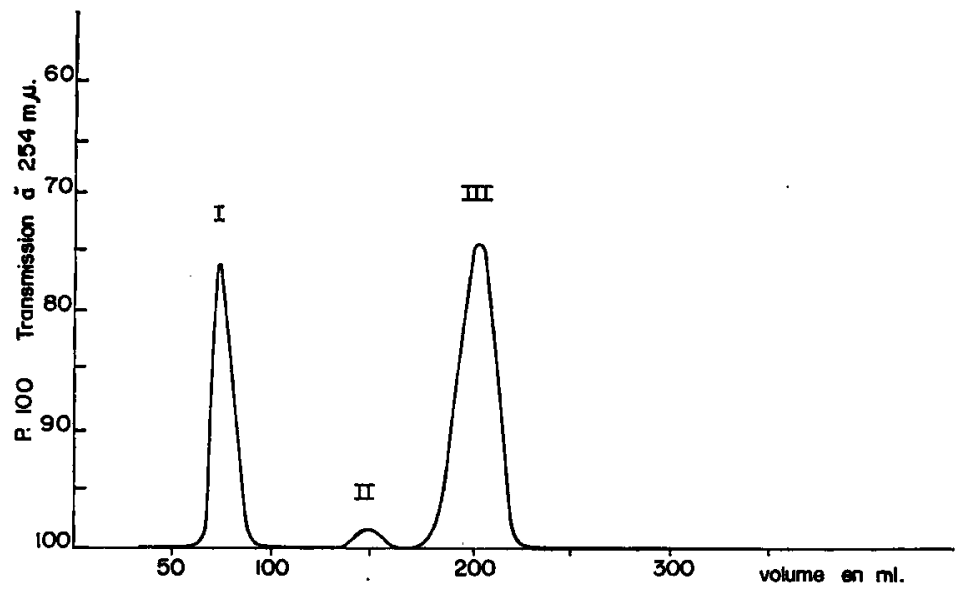

Fig. I . Fractionnement des pseudouridines sur Dowex $1 \times 8$, formate

sous forme chlorure. Les colonnes, $0,9 \times \mathrm{I} 6 \mathrm{~cm}\left(\mathrm{HCOO}^{-}\right)$et $0.9 \times \mathrm{I} 2 \mathrm{~cm}\left(\mathrm{Cl}^{-}\right)$, sont lavées à l'ammoniaque $0,2 \mathrm{~N} ; 1$ 'échantillon, 60 à $120 \mathrm{DO}_{260} \mathrm{pH} 7$, est adsorbé dans $5 \mathrm{ml}$ d'ammoniaque à $\mathrm{pH}$ II. L'élution est réalisée selon le cas par le formate d'ammonium $0,04 \mathrm{M}, \mathrm{pH} 8,6$, ou le chlorure d'ammonium $0,02 \mathrm{M}, \mathrm{pH}$ Io,6. 
Les spectres ultraviolets sont déterminés à trois $\mathrm{pH}$ contre un blanc approprié. Il est tenu compte de l'effet de dilution s'il y a lieu. La valeur de l'absorption au maximum à $\mathrm{pH}$ acide est, par convention, égale à une unité de densité optique. Quelle que soit la forme ionique de l'échangeur, le chromatogramme montre deux pics

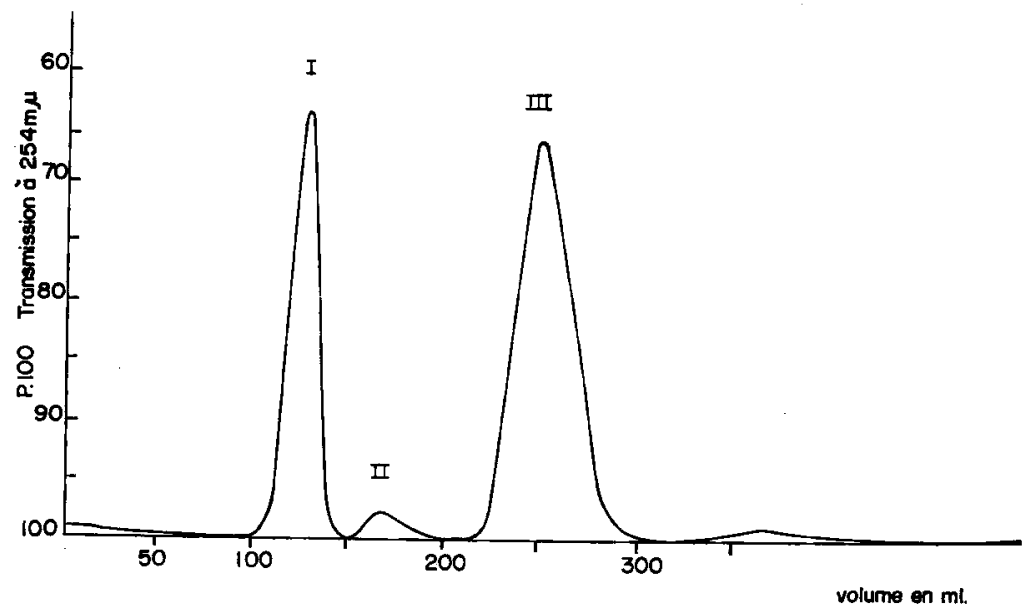

FIFG. 2 - Fractionnement des pseudouridines sur Dowex $1 \times 8$, chlorure

majeurs largement séparés, ainsi qu'un pic intermédiaire accessoire (fig. I et 2). De plus, l'utilisation de résine sous forme chlorure met en évidence une trace d'un constituant élué après le sommet III. Les spectres ultraviolets attestent dans les deux cas, que le pic I est la pseudouridine B et le pic III la pseudouridine C.

Nous présentons (fig. 3) les spectres obtenus après fractionnement sur Dowex I Cl-. Dans ce cas chaque sommet a été, au préalable, purifié sur une colonne d'amberlite I. R. A. I20 $\mathrm{H}^{+}$, pour éliminer l'effet tampon du chlorure d'ammonium. La faible quantité de matériel disponible ne nous a pas permis d'identifier avec certitude le pic II (fig. 2), mais les valeurs des rapports caractéristiques $\frac{\mathrm{DO}_{\max } \mathrm{pH} \text { I2 }}{\mathrm{DO}_{\max } \overline{\mathrm{pH}}-7}$

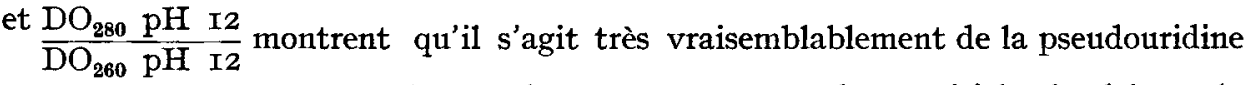
$\mathrm{A}_{s}$. Les sommets $\mathrm{I}, \mathrm{II}, \mathrm{III}$, représentent $99 \mathrm{p}$. Ioo du matériel adsorbé sur la colonne. Leur importance relative estimée par la densité optique à $260 \mathrm{~m} \mu$ à $\mathrm{pH} 7$, est la suivante :
I. Pseudouridine B, 3r p. Ioo,
II. Pseudouridine As, 2,54 p. Ioo
III. Pseudouridine C, 65,I7 p. I0o

Au cours des dernières années, diverses méthodes de fractionnement des isomères de la pseudouridine ont été proposées.

Dans la technique décrite par CoHN (I960) et modifiée par ShapIRo et ChamBERS (I96I) la séparation est obtenue en jouant sur l'aptitude plus ou moins grande de ces corps à former des complexes avec les ions borate. Cependant, la résolution entre les formes $A_{f}$ et $A_{s}$ n'est pas totale. Récemment, OFENGAND et SCHAFFER (I965) 
ont montré qu'il était possible, par chromatographie en couche mince sur cellulose, de réaliser une bonne séparation de trois des isomères de la pseudouridine. La technique que nous avons décrite nous a permis d'obtenir des résultats analogues. Il est intéressant de constater que l'analyse d'un échantillon de même provenance par des procédés différents, aboutit à des conclusions identiques quant à la proportion relative des divers isomères dans le mélange. Nous ne pouvons affirmer toutefois que des traces de pseudouridine $A_{f}$ ne contaminent pas l'un ou l'autre des sommets.
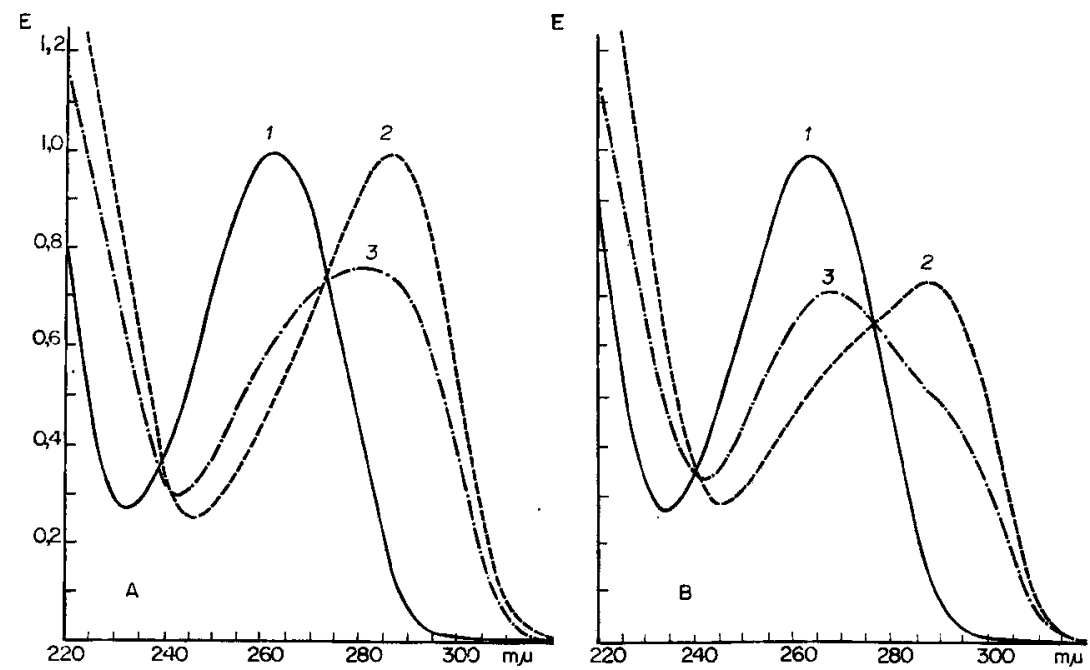

FIG. 3 .- Spectres d'absorption en lumière ultraviolelte de la pseudouridine $C(A)$,

$$
\begin{array}{lll}
\text { I. } \mathrm{pH} 2 ; & \text { 2. } \mathrm{pH} \text { II,90; } 3 \cdot \mathrm{pH} 9,40
\end{array}
$$

CoHs (I96o) signale que la pseudouridine C et l'uridine se séparent mal sur colonne d'échangeur d'anions. Pourtant, en utilisant la résine sous forme chlorure à $\mathrm{pH}$ Io,6, nous avons constaté que l'uridine est éluée très largement après la pseudouridine $\mathrm{C}$ et est immédiatement suivie de la ribothymidine. Ce système peut être utilisé dans le dosage des divers nucléosides uridyliques provenant de l'hydrolyse des ARN de transfert.

$$
\text { Reçu pour publication en avril } \mathrm{1967} \text {. }
$$

\section{SUMMARY}

ISOMER ISOLATION OF PSEUDOURIDINE

A pseudouridine sample (Calbiochem) was fractionated by simple chromatographic processes, into three isomers, viz: C, B and As. identified by ultra-violet spectrophotometry.

Our findings are in definite accordance with the literature.

\section{RÉFÉRENCES BIBLIOGRAPHIQUES}

ConN W. E., I960. Pseudouridine, a carbon-carbon linked ribonucleoside in ribonucleic acids : isolation structure, and chemical characteristics. J. Biol. Chem., 285, 1488-1498.

Ofengand J., Schaefer H., 1965. On the ionization constant of 5-ribosyluracil. Biochemistry, 4, 28322835 .

Shapiro R., Chambers R. W., Ig62. Synthesis of pseudouridine. J. Am. Chem. Soc., 83, 3920-3921. 